\title{
Desynchronization of coupled phase oscillators, with application to the Kuramoto system under mean-field feedback
}

\author{
Alessio Franci, Elena Panteley, Antoine Chaillet and Françoise Lamnabhi-Lagarrigue
}

\begin{abstract}
This note introduces two notions of desynchronization for interconnected phase oscillators by requiring that phases drift away from one another either at all times or in average. It provides a characterization of each of these two notions based on the grounded variable associated to the system, and relates them to a classical notion of instability valid in Euclidean spaces. An illustration is provided through the Kuramoto system, which is shown to be desynchronizable by proportional mean-field feedback.
\end{abstract}

\section{INTRODUCTION}

While most control applications aim at making a dynamical system converge to some prescribed behavior, control theory may sometimes be used to induce disorder in the dynamics. For instance, the possibility of disordering the output response of a system finds application in fluid mixing, optimization of abrasive machines, secure communication, heartbeat regulation, electromagnetic interference reduction, electrical load regulation, or acoustic noise attenuation. These applications have motivated the development of chaotification control laws, also called anti-control [28], [13], [5], [6]. For the particular case of interconnected agents, inducing more disorder in the dynamics results in the desynchronization of the involved agents. The development of desynchronizing control laws for coupled phaseoscillators has recently found application in the treatment of neurological diseases, cf. e.g. [11], [4], [27], [22].

While desynchronization owns quite an intuitive meaning, its formal definition is not straightforward. One way of guaranteeing sufficient disorder in a network of oscillators is to induce chaos in the incremental dynamics of their outputs (i.e. the dynamics ruling the phase differences of each pair of oscillators). This is the approach followed by chaotification techniques [28], [13], [5], [6]. However, chaos may be too strong a requirement in some particular applications and most anti-control techniques may require too much knowledge on the oscillators state to be practically implemented.

On the other hand, simply guaranteeing that phases are not synchronized is not enough in most practical applications. To see this, consider a pair of oscillators whose phases difference,

The research leading to these results has received funding from the European Union Seventh Framework Programme [FP7/2007-2013] under grant agreement n257462 HYCON2 Network of excellence, and by the French CNRS through the PEPS project TREMBATIC.

A. Franci is with Univ. Paris Sud 11 - L2S Supélec. 3, rue Joliot Curie, 91192 Gif sur Yvette, France alessio.francills.supelec.fr

E. Panteley is with CNRS - L2S, same address panteley@ls.s.supelec. fr

A. Chaillet is with Univ. Paris Sud 11 - L2S - EECI - Supélec, same address chailleteieee.org.

F. Lamnabhi-Lagarrigue is with CNRS - L2S - EECI, same address lamnabhi@lss.supelec.fr although not constant, remains at all times in a small neighboorhood of a given value. In this case, all classical definitions of synchronization are violated as the oscillators are neither phase synchronized [25], nor phase-locked [11] or frequencysynchronized [3] (as their phases difference is not constant). Nevertheless, for practical concerns, such a system cannot be considered as desynchronized since the phases difference remains "almost constant" at all times. In fact, such a situation would rather correspond to "approximative synchronization" as defined in [3]. In a nutschell, desynchronization is not simply the negation of synchronization.

In the textbook [20], and references therein, desynchronization is informally intended as the absence of approximate synchronization. This requirement translates in asking that the phase difference between two oscillators grows unbounded when lifted to the real line. The existence of unbounded trajectories is also the problem treated in [19]. As we show in the sequel, asking that the phase-difference is unbounded may not suffice to exclude asymptotic synchronization either.

The objective of this paper is then to define desynchronization in a rigorous manner for general networks of interconnected phase oscillators, and to provide a geometric and topological interpretation of this property by linking it to existing concepts of instability [18]. Roughly speaking, a pair of oscillators will be considered as desynchronized if their phases are permanently drifting away. Since this concept (referred to as strong desynchronization in Section II) is quite demanding due to the requirement of all-time phase drift, we also propose a relaxed notion, called practical desynchronization, that imposes phase drift only in average over a given time window (Secion III). For each of these properties, we propose a characterization inolving the grounded variables of the system, i.e. the differences between the oscillators' phases and their mean. These concepts are illustrated in Section IV through the Kuramoto system [16], for which we show that a proportional mean-field feedback can induce desynchronization.

Notation. Given $N \in \mathbb{N}_{\geq 1}, I_{N}$ denotes the $N \times$ $N$ indentity matrix, $\overrightarrow{1}_{N}$ is the $N$-dimesional vector whose entries are all $1, \mathrm{~T}^{N}$ denotes the $N$-torus and $\mathbb{N}_{N}^{\neq}:=$ $\left\{(i, j) \in\{1, \ldots, N\}^{2}: i \neq j\right\}$. Given $x \in \mathbb{R}^{n},|x|$ denotes the Euclidean norm of $x$. Given $x \in \mathbb{R}^{n}$ and $r \geq 0$, we denote by $\mathcal{B}_{r}(x)$ the closed ball of radius $r$ centered at $x$. Given $i \in\{1, \ldots, N\}, e_{i} \in \mathbb{R}^{N}$ is the vector with only zero entries, except the $i$-th which is equal to 1 . Given $x \in \mathbb{R}^{N}, x^{\perp}:=$ $\left\{z \in \mathbb{R}^{N}: x^{\top} z=0\right\}$. The solution of a system $\dot{x}=f(x, t)$ starting at $x_{0} \in \mathbb{R}^{n}$ at time $t_{0} \in \mathbb{R}$ is denoted by $x\left(\cdot ; t_{0}, x_{0}\right)$ everywhere it exists. Given a set $U \subset \mathbb{R}^{n}, x\left(t ; t_{0}, U\right):=$ $\left\{x\left(t ; t_{0}, x_{0}\right): x_{0} \in U\right\} . \mu$ denotes the Lebesgue measure on 
the set under consideration.

\section{DESYNCHRONIZATION AND ITS CHARACTERIZATION}

The dynamics of a network of coupled nonlinear phase oscillators can be expressed as

$$
\dot{\theta}=F(\theta, t),
$$

where $F: \mathrm{T}^{N} \times \mathbb{R} \rightarrow \mathbb{R}^{N}$ satisfies the Caratheodory conditions and $F(\cdot, t)$ is locally Lipschitz for each $t \in \mathbb{R}$. Since $F(\cdot, t)$ is defined on a compact space and it is locally Lipschitz, it is also bounded and globally Lipschitz. This ensures, together with the Caratheodory conditions, existence and unicity of the solution [14, Theorems 3.1 and 5.1] and forward completeness of (1). Each component $\theta_{i}, i=1, \ldots, N$, of $\theta$ is the phase of the oscillator $i$. The function $F$ describes both the internal dynamics of each oscillator and the coupling between different oscillators. This class of systems encompasses the phase oscillators studied in [4], of which the Kuramoto system [16] is probably the most famous representative (cf. Section IV for a deeper analysis).

\section{A. Definitions and first properties}

A pair $(i, j) \in \mathbb{N}_{N}^{\neq}$of coupled oscillators (1) undergoes frequency synchronization [3] if, given $t_{0} \in \mathbb{R}$ and $\theta_{0} \in \mathrm{T}^{N}$,

$$
\left|\dot{\theta}_{i}(t)-\dot{\theta}_{j}(t)\right|=0, \quad \forall t \in \mathbb{R},
$$

where $\theta_{i}(\cdot):=\theta_{i}\left(\cdot ; t_{0}, \theta_{0}\right)$ and similarly for $\theta_{j}(\cdot)$. This relation guarantees a constant phase difference between the oscillators $i$ and $j$, which is also referred to as phase-locking. A particular case of phase-locking is when this phase difference is zero, thus making $\theta_{i}(t)$ and $\theta_{j}(t)$ equal at all times. This stronger property is referred to as phase synchronization. When these properties hold asymptotically (i.e. as time goes to infinity), we refer to these properties as asymptotic phase-locking and asymptotic synchronization respectively [25], [20].

Asymptotic phase-locking is guaranteed (at least locally) if an asymptotically stable fixed point exists for the incremental dynamics ruling $\theta_{i}-\theta_{j}$. In the presence of exogenous disturbances or unmodelled dynamics, this asymptotically stable fixed point may present some robustness properties. We speak in this case of practical phase-locking [11], which can be formally characterized as ${ }^{1}$

$$
\left|\theta_{i}(t)-\theta_{j}(t)-\delta_{i j}\right| \leq \varepsilon_{i j}, \quad \forall t \in \mathbb{R},
$$

where $\delta_{i j} \in[0 ; 2 \pi)$ and $\varepsilon \geq 0$. When (3) holds only for a subset of pairs of oscillators, it is also referred to as partial entrainment [1]. Since $\theta \in \mathrm{T}^{N}$, the above constraint is trivially satisfied if $\varepsilon$ is greater than $\pi$. On the other hand, for small values of $\varepsilon$, the condition (3) imposes that the phase difference between oscillators $i$ and $j$, while not remaining constant, exhibit small oscillations around some constant values $\delta_{i j}$.

For desynchronization to have a practical relevance in most applications, it must exclude the two situations described by (2) and (3) and their asymptotic counterparts. Simply asking that the phase difference between two oscillators becomes unbounded when lifted on the real line may not be enough. Consider for instance the non-autonomous dynamics $\dot{\theta}_{ \pm}=\omega \pm \frac{\delta \omega}{t}$.

\footnotetext{
${ }^{1}$ We stress that the constant $\delta_{i j}$ and $\varepsilon_{i j}$ may depend on the inital conditions $\left(t_{0}, \theta_{0}\right)$.
}

Then $\theta_{+}(t)-\theta_{-}(t)=2 \delta \omega \ln (t)$, which grows unbounded, yet $\lim _{t \rightarrow \infty} \dot{\theta}_{+}(t)-\dot{\theta}_{-}(t)=0$, meaning that asymptotic phaselocking is achieved. Another natural requirement to make sure that the system is desynchronized is then to ask that the phases of oscillators $i$ and $j$ permanently drift away from one another, i.e. $\left|\dot{\theta}_{i}(t)-\dot{\theta}_{j}(t)\right|>0$, for all $t \in \mathbb{R}$. However, this requirement alone may not be enough either, since, for example, asymptotic synchronization, that is $\lim _{t \rightarrow \infty} \dot{\theta}_{i}(t)-\dot{\theta}_{j}(t)=0$, may satisfy it (if the convergence is achieved in infinite time only). For a pair of oscillators to be desynchronized, we therefore ask that the relative drift be uniformly bounded away from zero. This requirement ensures that the considered oscillators have their phases mutually drifting at all times and keeping on evolving in the torus with uniformly non-zero frequency difference. These conditions can be cast in a compact form in the following definition.

Definition 1 (Strong desynchronization) A pair $(i, j) \in \mathbb{N}_{N}^{\neq}$ of oscillators is said to be strongly desynchronized for (1) if there exists $\Omega_{i j}>0$ such that, for all $\theta_{0} \in \mathrm{T}^{N}$ and all $t_{0} \in \mathbb{R}$,

$$
\left|\dot{\theta}_{i}\left(t ; t_{0}, \theta_{0}\right)-\dot{\theta}_{j}\left(t ; t_{0}, \theta_{0}\right)\right| \geq \Omega_{i j}, \quad \forall t \in \mathbb{R} .
$$

Given $m \in\left\{1, \ldots, \frac{N(N-1)}{2}\right\}$, the network of coupled phase oscillators (1) is said to be $m$-strongly desynchronized if it contains $m$ distinct pairs of desynchronized oscillators. If $m=$ $\frac{N(N-1)}{2}$ then (1) is said to be completely strongly desynchronized.

Definition 1 satisfies two basic requirements: 1) it excludes synchronization and practical synchronization, also asymptotically; 2) it is naturally satisfied by an ensemble of uncoupled oscillators, provided the natural frequencies are not identical. However, we stress that Definition 1 does not exclude $p: q$ resonances $^{2}$ with $p \neq q$.

In order to give a geometrical interpretation of this property, we introduce the grounded variable $\psi \in \mathbb{R}^{N}$ associated to (1). Given some $\theta_{0} \in \mathrm{T}^{N}$ and some $t_{0} \in \mathbb{R}$, the evolution of $\psi$ is defined as

$$
\begin{aligned}
\dot{\psi}\left(t ; t_{0}, \theta_{0}\right) & :=\left(I_{N}-\frac{1}{N} \overrightarrow{1}_{N} \overrightarrow{1}_{N}^{\top}\right) \dot{\theta}\left(t ; t_{0}, \theta_{0}\right), \forall t \in \mathbb{R} \\
\psi\left(t_{0} ; t_{0}, \theta_{0}\right) & =\theta_{0}
\end{aligned}
$$

which constitutes a non-autonomous dynamics on $\mathbb{R}^{N}$. We refer to (5) as the grounded dynamics of (1). We stress that (5) could have been equivalently defined on either $\mathbb{R}^{N}$ or $\mathrm{T}^{N}$. Indeed, since $\dot{\theta}\left(t, t_{0}, \theta_{0}\right) \in \mathbb{R}^{N}$, for all $t, t_{0} \in \mathbb{R}$ and all $\theta_{0} \in \mathrm{T}^{N}$, (5) induces a well defined non-autonomous vector field on both $\mathbb{R}^{N}$ and $\mathrm{T}^{N}$. For future convenience we define here (5) as a dynamical system on $\mathbb{R}^{N}$. Noticing that $\frac{1}{N} \overrightarrow{1}_{N}^{\top} \dot{\theta}=\frac{1}{N} \sum_{i=1}^{N} \dot{\theta}_{i}$, this dynamics describes the evolution of the system (1) in a moving reference frame with speed equal to the instantaneous mean frequency $\frac{1}{N} \sum_{j=1}^{N} \dot{\theta}_{j}(t)$. This implies that $\dot{\psi}(t) \in \overrightarrow{1}_{N}^{\perp} \subset$ $\mathbb{R}^{N}$, for all $t \in \mathbb{R}$, where $\psi(\cdot):=\psi\left(\cdot ; t_{0}, \theta_{0}\right)$, that is the grounded dynamics has zero mean-drift, and $\overrightarrow{1}_{N}^{\top} \psi(t) \equiv \overrightarrow{1}_{N}^{\top} \theta_{0}$. In addition, it is possible to show that asymptotic phase-locking

\footnotetext{
${ }^{2} \mathrm{~A}$ pair of oscillator is said to be in a $p: q$ resonance, if the difference $\left|p \theta_{i}-q \theta_{j}\right|$ lifted to the real line remains bounded for all time, for some $p, q \in \mathbb{N}_{>0}$.
} 
of (1) corresponds to the existence of an asymptotically stable set for (5) (cf. e.g. [15]).

\section{B. Complete instability}

We introduce here the topological concept that serves as the basis of the characterization of desynchronization. This concept pertains to non-autonomous dynamical systems of the form

$$
\dot{x}=G(x, t),
$$

where $G: \mathbb{R}^{n} \times \mathbb{R} \rightarrow \mathbb{R}^{n}$ satisfies the Caratheodory conditions, and $G(\cdot, t)$ is continuous and locally Lipschitz, which ensures existence and unicity of the solution [14, Theorems 3.1 and 5.1].

Definition 2 (Complete instability, [18]) The dynamical system (6) is said to be completely unstable if all its points are wandering, that is for all $x_{0} \in \mathbb{R}^{n}$ and $t_{0} \in \mathbb{R}$, there exists a neighborhood $U$ of $x_{0}$ and a time $T>0$, such that $x\left(t ; t_{0}, U\right) \cap U=\emptyset$ for all $t \geq T+t_{0}$.

Complete instability can be considered as the complementary of asymptotic stability. Indeed, complete instability implies that, given any point, one can find a sufficiently small neighborhood around it, such that, after a sufficiently long time, the trajectories of the system leave the neighborhood and never go back in. The above definition is of no relevance to systems evolving in a compact space, as in this case the $\alpha$ - and $\omega$-limit sets are always non-empty ${ }^{3}[2]$.

In the following lemma we give a sufficient condition for (6) to be completely unstable.

Lemma 1 (Sufficient condition for complete instability) Suppose that (6) is forward complete. Suppose moreover that there exists a vector $\alpha \in \mathbb{R}^{n}$ and a constant $\bar{\alpha}>0$ such that, for all $x_{0} \in \mathbb{R}^{n}$ and $t_{0} \in \mathbb{R}$, the solution of (6) satisfies

$$
\alpha^{\top} G\left(x\left(t ; t_{0}, x_{0}\right), t\right) \geq \bar{\alpha}, \quad \forall t \in \mathbb{R} .
$$

Then (6) is completely unstable.

Proof of Lemma 1. From the assumption of the lemma it simply holds by integration that

$$
\left.\alpha^{\top}\left(x\left(t ; t_{0}, x_{0}\right), t\right)-x_{0}\right) \geq \bar{\alpha}\left(t-t_{0}\right) .
$$

Recalling that, for all $y \in \mathbb{R}^{n},|\alpha||y| \geq \alpha^{\top} y$, (7) implies that

$$
\left.\mid x\left(t ; t_{0}, x_{0}\right), t\right)-x_{0} \mid \geq \frac{\bar{\alpha}}{|\alpha|}\left(t-t_{0}\right) \text {. }
$$

Given $T>0$, consider the neighborhood $U$ of $x_{0}$ defined as $U:=\mathcal{B}_{r_{0}}\left(x_{0}\right)$, where $r_{0}:=\frac{\bar{\alpha} T}{4|\alpha|}$. From (8) it follows that $\left.x\left(t ; t_{0}, x_{0}\right), t\right) \notin U$ for all $t \geq \frac{T}{2}+t_{0}$, which ends the proof. $\square$

\footnotetext{
${ }^{3}$ The $\omega$ - (resp. $\alpha$-) limit set of a point $x_{0}$ is the union of all the points $\bar{x}$ for which there exists an increasing (resp. decreasing) and unbounded sequence of time instants $\left\{t_{n}\right\}_{n \in \mathbb{N}} \subset \mathbb{R}$ such that $\lim _{n \rightarrow \infty} x\left(t_{n} ; t_{0}, x_{0}\right)=\bar{x}$
}

\section{Desynchronization as complete instability}

Based on the considerations above, we now state the following theorem, which gives a geometrical and topological characterization of strong desynchronization in the sense of Definition 1.

Theorem 1 (Characterization of strong desynchronization) There exists a pair of strongly desynchronized oscillators $\theta_{i}, \theta_{j}$, $(i, j) \in \mathbb{N}_{N}^{\neq}$for the system (1), if and only if there exists a constant $\bar{\alpha}>0$, such that, for all $\theta_{0} \in T^{N}$ and $t_{0} \in \mathbb{R}$, the grounded dynamics (5) satisfies

$$
\dot{\psi}\left(t ; t_{0}, \theta_{0}\right)^{\top}\left(e_{i}-e_{j}\right) \geq \bar{\alpha}, \quad \forall t \in \mathbb{R},
$$

along the solutions of (1). In particular, if the pair $(i, j)$ is strongly desynchronized, then the grounded dynamics (5) associated to (1) is completely unstable.

Proof of Theorem 1. Necessity: Assume that the pair $(i, j)$ is strongly desynchronized. Then there exists a constant $\Omega_{i j}>0$ such that, given any $\theta_{0} \in \mathrm{T}^{N}$ and $t_{0} \in \mathbb{R}$, it holds that $\mid \dot{\theta}_{i}(t)-$ $\dot{\theta}_{j}(t) \mid \geq \Omega_{i j}$ for all $t \in \mathbb{R}$, where $\theta(\cdot):=\theta\left(\cdot ; t_{0}, \theta_{0}\right)$. Without loss of generality, we can pick $i, j$ in such a way that $\dot{\theta}_{i}(t)-$ $\dot{\theta}_{j}(t) \geq \Omega_{i j}$ for all $t \in \mathbb{R}$ (otherwise, just flip the indexes $i$ and $j$ ). Then, it holds from (5) that, for all $t \in \mathbb{R}$,

$$
\dot{\psi}(t)^{\top}\left(e_{i}-e_{j}\right)=\dot{\psi}_{i}(t)-\dot{\psi}_{j}(t)=\dot{\theta}_{i}(t)-\dot{\theta}_{j}(t) \geq \Omega_{i j},
$$

where $\psi(\cdot):=\psi\left(\cdot ; t_{0}, \theta_{0}\right)$. In particular, the solutions of (5) integrated along (1) satisfy $\dot{\psi}(t)^{\top}\left(\hat{e}_{i}-\hat{e}_{j}\right) \geq \Omega_{i j}$. The necessity part is then proved by picking $\bar{\alpha}=\Omega_{i j}$.

Sufficiency: Given $\theta_{0} \in \mathrm{T}^{N}$ and $t_{0} \in \mathbb{R}$, let (9) hold for some $\bar{\alpha}>0$. Then, for all $t \in \mathbb{R}$, it holds that

$$
\bar{\alpha} \leq \dot{\psi}(t)^{\top}\left(e_{i}-e_{j}\right)=\dot{\psi}_{i}(t)-\dot{\psi}_{j}(t)=\dot{\theta}_{i}(t)-\dot{\theta}_{j}(t) .
$$

The sufficiency part is then proved by picking $\Omega_{i j}=\bar{\alpha}$.

The rest of the statement follow by Lemma 1.

Theorem 1 highlights two properties of desynchronized dynamical systems: one geometrical, and the other topological. The first one, geometrical property, is contained in (9). It states that the grounded dynamics (5) is uniformly drifting away along the direction given by $e_{i}-e_{j} \in \overrightarrow{1}_{N}^{\perp}$. If we plug the meandrift back in, and project the resulting dynamics on the torus (to recover the original phase dynamics (1)), this means that in the $\left(\theta_{i}, \theta_{j}\right)$ sub-torus the trajectories of (1) are (locally) uniformly drifting away from the synchronization sub-manifold $\mathcal{D}_{i j}:=\left\{\theta \in \mathrm{T}^{N}: \theta_{i}=\theta_{j}\right\}$ (Figure 1). The topological characterization comes directly from the second part of the statement. In particular the grounded dynamics associated to a desynchronized system satisfies the complete instability property of Definition 2. We point out that this characterization complements the one associated to phase-locking, that is the asymptotic stability of the grounded dynamics.

Given $m \in\left\{1, \ldots, \frac{N(N-1)}{2}\right\}$, the following corollary, which is a direct consequence of Theorem 1 , gives a characterization of $m$-strong desynchronization and therefore of complete strong desynchronization. We stress that the above geometrical interpretation (Figure 1) extends to all the $m$ pairs of strongly desynchronized oscillators. 


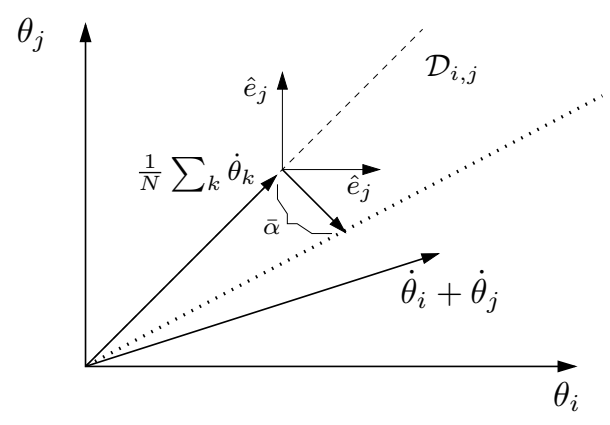

Fig. 1. Geometric interpretation of desynchronization

Corollary 1 (Characterization of $m$-strong and complete strong desynchronization) Given $m \in\left\{1, \ldots, \frac{N(N-1)}{2}\right\}$, if (1) is m-strongly desynchronized then its associated grounded dynamics (5) is completely unstable.

\section{PRACTICAL DESYNCHRONIZATION}

\section{A. Definition}

For particular applications, strong desynchronization may appear to be a too demanding requirement. For example, in electrical treatment of neurological diseases, only the average rate of discharge of the neurons is of interest [26], [24], [17], [21], [23]. More generally, the presence of exogenous disturbances, small coupling, or unmodelled dynamics may let the requirement of Definition 1 be too restrictive. The permanent phase drift imposed in Definition 1 impedes the instantaneous frequencies to be equal even on short time intervals. Intuitively, such a frequency similarity would not affect the overall desynchronization if it happens sufficiently rarely. Hence, we relax that definition by replacing the pointwise inequality (4) by the less restrictive assumption that the difference of frequencies be bounded from below in average, uniformly over some moving window of length $T$. This situation can be considered as the opposite of practical synchronization [11].

Definition 3 (Practical Desynchronization) A pair $(i, j) \in$ $\mathbb{N}_{N}^{\neq}$of oscillators is said to be practically desynchronized for (1) if there exists $\Omega_{i j}, T_{i j}>0$ such that, for all $\theta_{0} \in \mathrm{T}^{N}$ and $t_{0} \in \mathbb{R}$,

$$
\frac{1}{T_{i j}}\left|\int_{t}^{t+T_{i j}}\left(\dot{\theta}_{i}\left(\tau ; t_{0}, \theta_{0}\right)-\dot{\theta}_{j}\left(\tau ; t_{0}, \theta_{0}\right)\right) d \tau\right| \geq \Omega_{i j},
$$

for all $t \in \mathbb{R}$. Given $m \in\left\{1, \ldots, \frac{N(N-1)}{2}\right\}$, the network of coupled phase oscillators (1) is said to be m-practically desynchronized if it contains $m$ distinct pairs of practically desynchronized oscillators. If $m=\frac{N(N-1)}{2}$ then (1) is said to be completely practically desynchronized.

In the following proposition we show that if (1) is timeinvariant, then uniformity of (10) in $\theta_{0}$ suffices to ensure its uniformity in time. This lets (10) be easier to check in practice (see also Theorem 2 below).

Proposition 1 Suppose that (1) is time-invariant. Given $T_{i j}, \Omega_{i j}>0$, assume that there exists a pair of oscillators $(i, j) \in \mathbb{N}_{N}^{\neq}$satisfying (10) for all $\theta_{0} \in T^{N}$, and for some $t=t^{*} \in \mathbb{R}$. Then (10) holds for all $t \in \mathbb{R}$.

Proof of Proposition 1 Since the dynamics (1) is time invariant we can pick, without loss of generality, $t^{*}=0$ in the statement of the proposition. Let $\theta_{0} \in \mathrm{T}^{N}$. Since (1) defines a smooth bounded dynamics, $\theta\left(t ; t_{0}, \theta_{0}\right)$ exists for all time. Fix any $t \in \mathbb{R}$, and let $\theta(t):=\theta\left(t ; t_{0}, \theta_{0}\right)$. The system (1) being time invariant, and since (10) holds uniformly in the initial conditions, it also holds that

$$
\begin{aligned}
& \frac{1}{T_{i j}}\left|\int_{t}^{t+T_{i j}}\left(\dot{\theta}_{i}\left(\tau ; t_{0}, \theta_{0}\right)-\dot{\theta}_{j}\left(\tau ; t_{0} \theta_{0}\right)\right) d \tau\right| \\
= & \frac{1}{T_{i j}} \mid \int_{0}^{T_{i j}}\left(\dot{\theta}_{i}\left(\tau ; t_{0}, \theta(t)\right)-\dot{\theta}_{j}\left(\tau ; t_{0}, \theta(t)\right) d \tau \mid\right. \\
\geq & \Omega_{i j} .
\end{aligned}
$$

Since $t \in \mathbb{R}$ is arbitrary, the proposition is proved.

\section{B. Characterization of practical desynchronization}

In order to extend the interpretation developped in Section II$\mathrm{C}$ to the case of practical synchronization, we introduce an averaged system associated to (1). Given any $T>0$, any $\theta_{0} \in \mathrm{T}^{N}$, and any $t_{0} \in \mathbb{R}$, the $T$-averaged system associated to (1) is defined as

$$
\begin{aligned}
\widehat{\left\langle\theta\left(t ; t_{0}, \theta_{0}\right)\right\rangle_{T}} & :=\frac{1}{T} \int_{t}^{t+T} \dot{\theta}\left(\tau ; t_{0}, \theta_{0}\right) d \tau, \\
\left\langle\theta\left(t_{0} ; t_{0}, \theta_{0}\right)\right\rangle_{T} & :=\theta_{0}
\end{aligned}
$$

for all $t \in \mathbb{R}$. The averaged system evolves with the average instantaneous frequency of the system (1) over a sliding time window of length $T$. We point out that, since $\frac{1}{T} \int_{t}^{t+T} \dot{\theta}\left(\tau ; t_{0}, \theta_{0}\right) d \tau \in \mathbb{R}^{N}$ for all $t \in \mathbb{R},(11)$ is a well defined non-autonomous dynamics on $T^{N}$.

The following lemma, whose proof is trivial and is omitted, shows that the practical desynchronization of (1) corresponds to the strong desynchronization of its averaged system.

\section{Lemma 2 (Desynchronization of the averaged system)}

There exists a pair $(i, j) \in \mathbb{N}_{N}^{\neq}$of practically desynchronized oscillators for the system (1), (that is $\theta_{i}, \theta_{j}$ satisfy (10) for some $\Omega_{i j}, T_{i j}>0$ ) if and only if the $T_{i j}$-averaged system (11) associated to (1), satisfies

$$
\left.\left|\widehat{\left\langle\theta_{i}\left(t ; t_{0}, \theta_{0}\right)\right.}\right\rangle_{T_{i j}}-\widehat{\left\langle\theta_{j}\left(t ; t_{0}, \theta_{0}\right)\right.}\right\rangle_{T_{i j}} \mid \geq \Omega_{i j}, \forall t \in \mathbb{R},
$$

that is $\left\langle\theta_{i}\right\rangle_{T_{i j}}$ and $\left\langle\theta_{j}\right\rangle_{T_{i j}}$ are strongly desynchronized.

At the light of the above lemma we are able to give a characterization of practical desynchronization. For all $T \geq 0$, the $T$-averaged grounded dynamics $\psi_{T} \in \mathbb{R}^{N}$ associated to (11) is given by

$$
\begin{aligned}
\dot{\psi}_{T}\left(t ; t_{0}, \theta_{0}\right) & :=\left(I_{N}-\frac{1}{N} \overrightarrow{1}_{N} \overrightarrow{1}_{N}^{\top}\right){\widehat{\left\langle\theta\left(t ; t_{0}, \theta_{0}\right)\right\rangle_{T}}} \\
\psi_{T}\left(t_{0} ; t_{0}, \theta_{0}\right) & =\theta_{0} .
\end{aligned}
$$

The following corollary, which is a direct consequence of Theorem 1 and Lemma 2, provides a complete characterization of practical desynchronization. 
Corollary 2 (Characterization of practical desynchronization) There exists a pair $(i, j) \in \mathbb{N}_{N}^{\neq}$of practically desynchronized oscillators for system (1) if and only if there exist some constants $\bar{\alpha}, T>0$, such that, for all $\theta_{0} \in T^{N}$ and all $t_{0} \in \mathbb{R}$, the $T$-averaged grounded dynamics (13) satisfies

$$
\dot{\psi}_{T}\left(t ; t_{0}, \theta_{0}\right)^{\top}\left(e_{i}-e_{j}\right) \geq \bar{\alpha}, \quad \forall t \in \mathbb{R}
$$

In particular, if the pair $(i, j)$ is practically desynchronized, the T-averaged grounded dynamics (13) associated to (1) is completely unstable.

\section{Desynchronization of the Kuramoto system THROUGH MEAN-FIELD FEEDBACK}

The Kuramoto system under mean-field feedback (MFF) appears as a simple model of coupled oscillators under the influence of a scalar MFF. In [11], [9] it was studied with the scope of efficient desynchronization of brain cells for the treatment of neurological diseases by electrical stimulation. In those references the following model is derived

$$
\begin{aligned}
\dot{\theta}_{i}= & \omega_{i}+\frac{1}{N} \sum_{j=1}^{N}\left(k_{i j}+\gamma_{i j}\right) \sin \left(\theta_{j}-\theta_{i}\right) \\
& -\frac{1}{N} \sum_{j=1}^{N} \gamma_{i j} \sin \left(\theta_{j}+\theta_{i}\right), \forall i=1, \ldots, N,
\end{aligned}
$$

where $\theta_{i}$ represents the phase of the oscillator $i$, the parameters $k_{i j}$ represent the interconnection gains, and $\gamma_{i j}$ are gains resulting from the application of the proportional mean-field feedback. See [11] and [9] for details.

It was shown in [11] that, for almost all interconnection topology and almost all value of the feedback gain, phaselocking is impossible under MFF. In the next theorem we show that practical desynchronization can actually be achieved by MFF. In other words, we give a sufficient condition to assure that a given couple of oscillators is practically desynchronized while the ensemble keeps on oscillating (Figure 2).

Theorem 2 (Practical desynchronization of the Kuramoto system under MFF) Suppose that there exists $i, j \in \mathbb{N}_{N}^{\neq}$, such that

$$
\begin{aligned}
& \Omega_{i j}:= \\
& \left|\omega_{i}-\omega_{j}\right|-\frac{1}{N} \sum_{h=1}^{N}\left|\gamma_{i h}+\gamma_{j h}\right|\left(\frac{\pi \nu}{2 \bar{\omega}}+\frac{\nu^{2}}{6 \bar{\omega}^{2}}\right)-\frac{1}{N} \sum_{h=1}^{N}\left|\varepsilon_{i h}+\varepsilon_{j h}\right| \\
& >0,
\end{aligned}
$$

where $\nu:=2 \max _{h=1, \ldots, N}\left(\left|\tilde{\omega}_{h}\right|+\frac{1}{N} \sum_{h^{\prime}=1}^{N}\left|\gamma_{h h^{\prime}}+\varepsilon_{h h^{\prime}}\right|\right)$, $\bar{\omega}:=\frac{1}{N} \overrightarrow{1}_{N}^{\top} \omega, \tilde{\omega}_{h}:=\omega_{h}-\bar{\omega}$, and $\varepsilon_{h h^{\prime}}:=k_{h h^{\prime}}+\gamma_{h h^{\prime}}$, for all $h, h^{\prime}=1, \ldots, N$.

Then the pair of oscillators $(i, j)$ is practically desynchronized.

The sufficient condition (16) can readily be used in practical applications to explicitly compute the minimum number of desynchronized pairs of oscillators. The term $\frac{1}{N} \sum_{h=1}^{N}\left|\gamma_{i h}+\gamma_{j h}\right|\left(\frac{\pi \nu}{2 \bar{\omega}}+\frac{\nu^{2}}{6 \bar{\omega}^{2}}\right)$ is small provided the mean natural frequency $\bar{\omega}$ is large. In the opposite case, one rather expects the mean-field feedback to block the oscillations, as

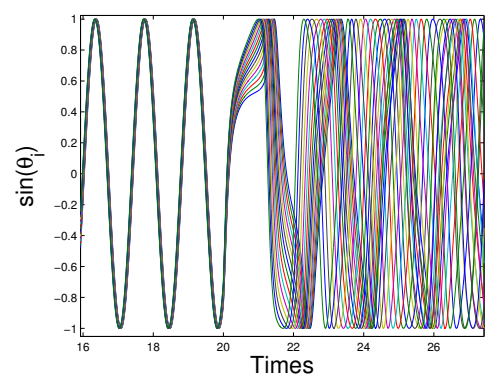

Fig. 2. Evolution of the phases of (17) for large natural frequencies when a proportional mean-field feedback with gain $\gamma=-2 k_{0}$ is switched on at time $t=20$. The mean-field feedback induces desynchronization.

described in [10], [12]. The term $\sum_{h=1}^{N}\left|\varepsilon_{i h}+\varepsilon_{j h}\right|$ guides the feedback gain design to obtain oscillators desynchronization by imposing to minimize the closed-loop diffusive coupling strength $k_{i j}+\gamma_{i j}$. In terms of the grounded dynamics associated to (18), Corollary 2 implies that its average system is completely unstable (Figure 3).

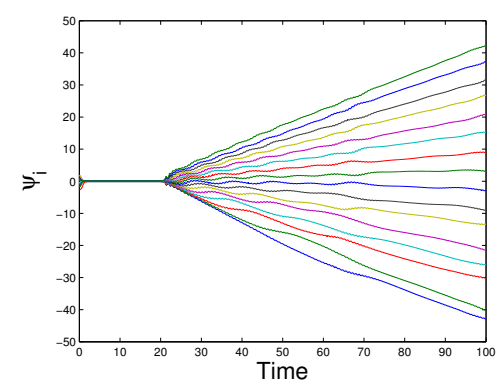

Fig. 3. Evolution of the grounded dynamics of (17) for large natural frequencies when a proportional mean-field feedback with gain $\gamma=-k_{0}$ is switched on at time $t=20$

In the case when the coupling is given by the all-to-all topology, and each oscillator contributes in the same way at the measured mean-field and receives the input with same intensity, the interconnection and feedback gains become $k_{i j}=k_{0}$ and $\gamma_{i j}=\gamma$, for all $i, j=1, \ldots, N$. In this case (15) reduces to

$$
\begin{aligned}
\dot{\theta}_{i}= & \omega_{i}+\frac{\left(k_{0}+\gamma\right)}{N} \sum_{j=1}^{N} \sin \left(\theta_{j}-\theta_{i}\right) \\
& -\frac{\gamma}{N} \sum_{j=1}^{N} \sin \left(\theta_{j}+\theta_{i}\right), \forall i=1, \ldots, N .
\end{aligned}
$$

The diffusive coupling term $\left(k_{0}+\gamma\right) \sum_{j=1}^{N} \sin \left(\theta_{j}-\theta_{i}\right)$ can be eliminated by choosing $\gamma=-k_{0}$, and (17) reduces to

$$
\dot{\theta}_{i}=\omega_{i}+\frac{k_{0}}{N} \sum_{j=1}^{N} \sin \left(\theta_{j}+\theta_{i}\right), \forall i=1, \ldots, N .
$$

Theorem 2 then relaxes in this case to the following corollary.

Corollary 3 (Practical desynchronization of the all-to-all Kuramoto system under MFF) Suppose that there exists $i, j \in$ $\mathbb{N}_{N}^{\neq}$, such that

$$
\Omega_{i, j}:=\left|\omega_{i}-\omega_{j}\right|-2 k_{0}\left(\frac{\pi \nu}{2 \bar{\omega}}+\frac{\nu^{2}}{6 \bar{\omega}^{2}}\right)>0,
$$


where $\nu:=2 \max _{h=1, \ldots, N}\left(\left|\tilde{\omega}_{h}\right|+k_{0}\right), \bar{\omega}:=\frac{1}{N} \overrightarrow{1}_{N}^{\top} \omega$, and $\tilde{\omega}_{h}:=\omega_{h}-\bar{\omega}$, for all $h=1, \ldots, N$.

Then the pair of oscillators $(i, j)$ is practically desynchronized.

Inequality (19) is always satisfied, provided that $\omega_{i} \neq \omega_{j}$ and $\bar{\omega}$ is sufficiently large (Figures 2 and 3 ). Indeed, the minimum coupling strength that ensures asymptotic phase-locking of (17) in the absence of mean-field feedback does not depend on the absolute magnitude of the natural frequencies, but only on their dispersion [8], [15], [7], [11]. One thus expects the value of $k_{0}$ guaranteeing phase-locking in the absence of MFF to be independent of $\bar{\omega}$.

\section{Proof of Theorem 2}

The whole proof is based on the following claim, whose proof follows from elementary trigonometry and is omitted for space reasons. The interested reader can find it in [10].

Claim 1 For all $\theta_{0} \in T^{N}$, the trajectory of (18) satisfies, for all $i, j=1, \ldots, N$,

$$
\frac{\bar{\omega}}{\pi}\left|\int_{0}^{\pi / \bar{\omega}} \sin \left(\theta_{i}(\tau)+\theta_{j}(\tau)\right) d \tau\right| \leq\left(\frac{\pi \nu}{2 \bar{\omega}}+\frac{\nu^{2}}{6 \bar{\omega}^{2}}\right) .
$$

Invoking Proposition 1 and Claim 1 , it follows that, for all $\theta_{0} \in$ $\mathrm{T}^{N}$, all $i, j \in \mathbb{N}_{N}^{\neq}$, and all $t \in \mathbb{R}$, the trajectory of (18) satisfies

$$
\begin{aligned}
& \frac{\bar{\omega}}{\pi}\left|\int_{t}^{t+\pi / \bar{\omega}}\left(\dot{\theta}_{i}(\tau)-\dot{\theta}_{j}(\tau)\right) d \tau\right| \\
\geq & \left|\omega_{i}-\omega_{j}\right| \\
& \quad-\sum_{l=i, j} \sum_{h=1}^{N} \frac{\gamma_{l h}}{N} \frac{\bar{\omega}}{\pi}\left|\int_{t}^{t+\pi / \bar{\omega}} \sin \left(\theta_{l}(\tau)+\theta_{h}(\tau)\right) d \tau\right| \\
& -\sum_{l=i, j} \sum_{h=1}^{N} \frac{\varepsilon_{l h}}{N} \frac{\bar{\omega}}{\pi}\left|\int_{t}^{t+\pi / \bar{\omega}} \sin \left(\theta_{l}(\tau)-\theta_{h}(\tau)\right) d \tau\right|, \forall t \geq 0 \\
\geq & \left|\omega_{i}-\omega_{j}\right|-\frac{1}{N} \sum_{h=1}^{N}\left|\gamma_{i h}+\gamma_{j h}\right|\left(\frac{\pi \nu}{2 \bar{\omega}}+\frac{\nu^{2}}{6 \bar{\omega}^{2}}\right)-\frac{1}{N} \sum_{h=1}^{N}\left|\varepsilon_{i h}+\varepsilon_{j h}\right| \\
> & 0,
\end{aligned}
$$

where the last inequality comes from assumption (16). Recalling Definition 3, this proves the theorem.

\section{REFERENCES}

[1] D. Aeyels and J. A. Rogge. Existence of partial entrainment and stability of phase locking behavior of coupled oscillators. Progress of Theoretical Physics, 112(6):921-942, 2004.

[2] N. P. Bhatia and G. P. Szegö. Stability theory of dynamical systems. Die Grundlehren der mathematischen Wissenschaften, Band 161. Springer-Verlag, New York, 1970.

[3] I.I. Blekhman, A.L. Fradkov, H. Nijmeijer, and A.Y. Pogromsky. On self synchronization and controlled synchronization. Syst. \& Contr. Letters, 31:299-305, 1997.

[4] E. Brown, P. Holmes, and J. Moehlis. Globally coupled oscillator networks. In Perspectives and problems in nonlinear science: A celebratory volume in honor of Larry Sirovich, pages 183-215, 2003.

[5] G. Chen. Chaotification via feedback: The discrete case. In Lecture Notes in Control and Information Sciences, volume 292. Springer, 2003.

[6] G. Chen and L. Yang. Chaotifying a continuous-time system near a stable limit cycle. Chaos, Solitons and Fractals, 15(2):245-253, 2003.
[7] N. Chopra and M. W. Spong. On exponential synchronization of Kuramoto oscillators. IEEE Trans. on Automat. Contr., 54(2):353$357,2009$.

[8] F. Dörfler and F. Bullo. Synchronization and transient stability in power networks and non-uniform Kuramoto oscillators. IEEE Trans. on Automat. Contr., 2011. (submitted).

[9] A. Franci, A. Chaillet, and S. Bezzaoucha. Toward oscillations inhibition by mean-field feedback in Kuramoto oscillators. In Proc. IFAC World Congress, Milan, Italy, August 2011.

[10] A. Franci, A. Chaillet, E. Panteley, and F. Lamnabhi-Lagarrigue. Desynchronization and inhibition of all-to-all interconnected $\mathrm{Ku}-$ ramoto oscillators by scalar mean-field feedback. Mathematics of Control, Signals, and Systems - Special Issue on large-scale nonlinear systems, 2011. Submitted.

[11] A. Franci, A. Chaillet, and W. Pasillas-Lépine. Existence and robustness of phase-locking in coupled Kuramoto oscillators under mean-field feedback. Automatica - Special Issue on Biology Systems, 47(6):1193-1202, 2010. Regular paper. Extended version available at: http://hal.archives-ouvertes.fr/hal-00526066/.

[12] A. Franci, A. Chaillet, and W. Pasillas-Lépine. Robustness of phaselocking between Kuramoto oscillators to time-varying inputs. In Proc. IFAC World Congress, Milan, Italy, August 2011.

[13] Y. Gao and K. Chau. Chaotification of permanent-magnet synchronous motor drives using time-delay feedback. In IEEE Annual Conf. of Industrial Electronics Soc., pages 762-766, 2002.

[14] J.K. Hale. Ordinary Differential equations. Interscience. John Wiley, New York, 1969.

[15] A. Jadbabaie, N. Motee, and M. Barahona. On the stability of the Kuramoto model of coupled nonlinear oscillators. Proc. American Control Conf., pages 4296-4301, 2004.

[16] Y. Kuramoto. Chemical Oscillations, Waves, and Turbulence. Springer, Berlin, 1984.

[17] J. Lopez-Azcarate, M. Tainta, M. C. Rodriguez-Oroz, M. Valencia, R. Gonzalez, J. Guridi, J. Iriarte, J. A. Obeso, J. Artieda, and M. Alegre. Coupling between beta and high-frequency activity in the human subthalamic nucleus may be a pathophysiological mechanism in Parkinsons disease. J. Neurosci., 30(19):6667-6677, 2010.

[18] V.V. Nemytskii and V.V. Stepanov. Qualitative theory of differential equations. Dover Publications, INC., New York. Reprint. Originally published: Princeton University Press, 1960

[19] R. Orsi, L. Praly, and I. Mareels. Sufficient conditions for the existence of an unbounded solution. Automatica, 37(10):1609-1617, 2001.

[20] A. Pikovsky, M. Rosenblum, and J. Kurths. Synchronization: A Universal Concept in Nonlinear Sciences. Cambridge Nonlinear Science Series, Cambridge, United Kingdom, 2001.

[21] D. Plenz and S. T. Kital. A basal ganglia pacemaker formed by the subthalamic nucleus and external globus pallidus. Nature, 400(6745):677-682, 1999.

[22] K. Pyragas, O. V. Popovich, and P. A. Tass. Controlling synchrony in oscillatory networks with a separate stimulation-registration setup. EPL, 80(4), 2008.

[23] M. Rosa, S. Marceglia, D. Servello, G. Foffani, L. Rossi, M. Sassi, S. Mrakic-Sposta, R. Zangaglia, C. Pacchetti C, M. Porta, and A. Priori. Time dependent subthalamic local field potential changes after DBS surgery in Parkinson's disease. Experimental Neurology, 222(2): 184-190, 2010

[24] S. V. Sarma, M. Cheng, Z. Williams, R. Hu, E. Eskandar, and E. N. Brown. Comparing healthy and Parkinsonian neuronal activity in subthalamic nucleus using point process models. IEEE Trans Biomed Eng., 57(6):1297-1305, 2010.

[25] S. H. Strogatz. From Kuramoto to Crawford: Exploring the onset of synchronization in population of coupled oscillators. Physica $D$, 143:1-20, 2000.

[26] J. Volkmann, M. Joliot, A. Mogilner, A. A. Ioannides, F. Lado, E. Fazzini, U. Ribary, and R. Llinás. Central motor loop oscillations in Parkinsonian resting tremor revealed by magnetoencephalography. Neurology, 46:1359-1370, 1996.

[27] O. V.Popovych, C. Hauptmann, and P. A. Tass. Desynchronization and decoupling of interacting oscillators by nonlinear delayed feedback. Internat. J. Bifur. Chaos, 16(7):1977-1987, 2006.

[28] H. Zhang, D. Liu, and Z. Wang. Controlling Chaos: Suppression, Synchronization and Chaotification. Communications and Control Engineering. Springer-Verlag, 2009. 